\title{
Correlação Entre as Propriedades Reológicas, Óticas e a Morfologia de Filmes Soprados de LLDPE/LDPE
}

\author{
Lilia M. Guerrini, Pedro I. Paulin Fo, Rosario E. S. Bretas \\ Departamento de Engenharia de Materiais, UFSCar
}

\section{Alessandro Bernardi \\ Braskem, Triunfo, $R S$}

Resumo: Neste trabalho correlações entre as propriedades reológicas e óticas e a morfologia de filmes soprados de polietileno linear de baixa densidade, LLDPE, e de suas blendas com $10 \%$ e $20 \%$ de polietileno de baixa densidade, LDPE, foram pesquisadas. Os filmes foram processados em uma sopradora industrial. As propriedades reológicas estudadas foram : a viscosidade em regime permanente de cisalhamento, $\eta(\dot{\gamma})$, a primeira diferença de tensões normais, $N_{1}(\dot{\gamma})$, o módulo complexo em cisalhamento, $\mathrm{G}^{*}(\omega)$ e a deformação recuperável, $\gamma_{\mathrm{r}}(\mathrm{t}) . \eta(\dot{\gamma})$ e $N_{1}(\dot{\gamma})$ foram medidos num reômetro de deformação controlada com cone e placa (taxas baixas) e num capilar (taxas elevadas); $\mathrm{G}^{*}$ $(\omega)$ e $\gamma_{\mathrm{r}}(\mathrm{t})$ foram medidos num reômetro de tensão controlada com placas paralelas. As propriedades óticas (brilho e opacidade) foram medidas num opacímetro. As morfologias superficiais dos filmes soprados foram analisadas por microscopia eletrônica de varredura e microscopia de força atômica. Uma correlação entre deformação recuperável, opacidade total e morfologia dos filmes das blendas de LLDPE/LDPE foi obtida: quanto maior a recuperação elástica, menor a opacidade total e menor o tamanho do cristalito. A adição do LDPE ao LLDPE aumentou a deformação recuperável das blendas, diminuindo as suas opacidades.

Palavras-chave: Filmes soprados, propriedades reológicas, propriedades óticas, polietileno linear de baixa densidade, polietileno de baixa densidade.

\section{Correlation Between Rheological and Optical Properties and the Morphology of Blown Films of LLDPE/LDPE}

Abstract: In this work, correlations between the rheological and optical properties and the morphology of blow films of blends of linear low density, LLDPE, and low density polyethylene, LDPE, were studied. The films were blown in an industrial film blowing line. Rheological properties of the blends, such as shear steady state viscosity, $\eta(\dot{\gamma})$, first normal stress difference, $N_{1}(\dot{\gamma})$, shear complex modulus, $\mathrm{G}^{*}(\omega)$ and recoverable strain, $\gamma_{\mathrm{r}}(\mathrm{t})$ were analyzed. $\eta(\dot{\gamma})$ and $N_{1}(\dot{\gamma})$ were measured in a strain controlled rheometer with cone and plate geometry (low shear rates) and in a capillary rheometer (hig shear rates); on the other hand, $G^{*}(\omega)$ and $\gamma_{\mathrm{r}}(t)$ were measured in a tension controlled rheometer with parallel plates geometry. The optical properties (specular gloss and haze) were also measured in standard equipment. The surface morphology of the blown films was analyzed by scanning electron and atomic force microscopes. A correlation between recoverable strain, total haze and surface morphology of the blown films was obtained: the higher the recoverable strain, the lower the total haze and the smaller the crystallites. The addition of the LDPE to the LLDPE increased the recoverable strain of the films, decreasing their haze.

Keyword: Blow films, rheological properties, optical properties, linear low density polyethylene, low density polyethylene.

\section{Introdução}

Filmes soprados são produzidos pela extrusão do polímero fundido, na forma de um tubo, através de uma matriz anelar, no centro da qual ar é injetado, inflando o tubo até este atingir um diâmetro maior ${ }^{[1]}$, como mostrado na Figura 1. Uma "bolha" é então formada, cujas paredes são estiradas na circunferência (pelo ar injetado) e na vertical, por rolos puxadores, ao mesmo tempo em que são resfriadas, conferindo então ao filme soprado uma orientação biaxial. Os parâmetros mais importantes deste processo são a razão de sopro, $B U R=R_{f} / R_{o}$, onde $R_{f}=$ raio final do filme soprado, $R_{0}=$ raio inicial do filme, a razão de estiramento $\mathrm{D}_{\mathrm{r}}=\mathrm{V} / \mathrm{V}_{\mathrm{o}}$, onde $\mathrm{V}=$ velocidade de puxamento e $\mathrm{V}_{\mathrm{o}}=$ velocidade na saída da matriz e a linha de congelamento, $\mathrm{Z}$. Acima de Z, considera-se que o polímero está solidificado, com a sua estrutura cristalina e orientação congeladas. Estes dois últimos parâmetros moleculares dependerão então das condições do fluxo, ou seja, das propriedades reológicas do polímero. Pode-se observar que existem três tipos de fluxos durante o sopro. Na matriz anelar, o fluxo é predominantemente de cisalhamento; entre a saída da matriz e o

Autor para correspondência: Rosario E. S. Bretas, DEMa, UFSCar, Caixa Postal 676, CEP: 13565-905, São Carlos, SP. E-mail: bretas@ power.ufscar.br 
início da "bolha", o fluxo é uma mistura de cisalhamento com fluxo elongacional. Até $\mathrm{Z}$, o fluxo é predominantemente elongacional. Na matriz, por exemplo, propriedades reológicas, como viscosidade em regime permanente de cisalhamento, $\eta(\dot{\gamma})$, e primeira diferença de tensões normais, $N_{1}(\dot{\gamma})$, onde $\dot{\gamma}$ é a taxa de cisalhamento, determinam a facilidade de escoamento do material e o inchamento do extrudado ${ }^{[2]}$; na região de transição, propriedades reológicas, como o reemaranhamento após término do fluxo de cisalhamento, medido pela deformação recuperável de cisalhamento, $\gamma_{\mathrm{r}}(\mathrm{t})^{[2]}$ e o módulo complexo em cisalhamento, $\mathrm{G}^{*}(\omega)=\mathrm{G}^{\prime}+\mathrm{i} \mathrm{G}^{\prime}$, onde $\mathrm{G}^{\prime}=$ módulo de armazenamento em cisalhamento e $\mathrm{G}$ "=módulo de perda em cisalhamento, determinam o tempo de relaxação do polímero e a estabilidade da bolha, respectivamente; finalmente, na região de fluxo elongacional, a viscosidade elongacional, $\bar{\eta}(\dot{\varepsilon})$, onde $\dot{\varepsilon}$ é a taxa de elongação, determina a quantidade de orientação molecular que pode ser conseguida.

O uso principal destes filmes é na área de embalagens. Conseqüentemente, eles devem ser transparentes para permitir a visualização da mercadoria pelo consumidor. Durante o processamento, torna-se necessário, então, otimizar as propriedades óticas destes filmes, tais como o brilho e as opacidades interna e superficial. O brilho é um fenômeno reflexivo medido num ângulo pré-determinado e, em geral, quanto mais lisa a superfície de um filme, maior o brilho desta. A opacidade superficial é conseqüência da rugosidade superficial, a qual, por sua vez, dependerá das condições de processo, tais como

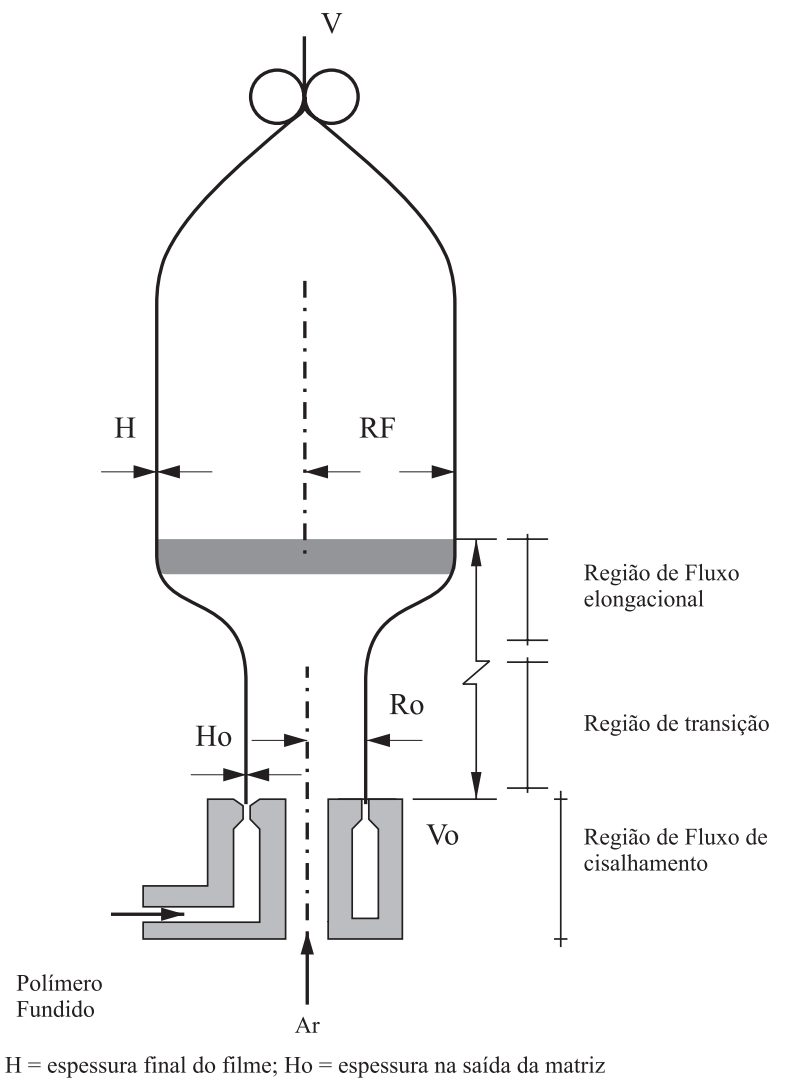

Figura 1. Esquema do processo de sopro de filmes ${ }^{[1]}$.
BUR, $D_{r}, Z$ e das propriedades reológicas do material. Já a opacidade interna éinfluenciada pelo arranjo morfológico das fases cristalinas. Por exemplo, se os tamanhos dos diâmetros dos esferulitos se aproximarem dos comprimentos de onda da luz visível, maior será o valor desta opacidade.

Dentre os polímeros mais utilizados para fazer filmes soprados, encontra-se o polietileno de baixa densidade, LDPE, o qual na forma de filme, possui boas propriedades óticas e boa processabilidade, porém baixa resistência mecânica. $\mathrm{O}$ contrário ocorre com o polietileno linear de baixa densidade, LLDPE, o qual, como filme possui propriedades óticas pobres, processabilidade difícil, mas boa resistência mecânica. Assim, é comum utilizar blendas destes dois polímeros para obter filmes com boa processabilidade, boas propriedades óticas e boa resistência mecânica.

Vários autores têm estudado a influência da adição de LDPE em LLDPE nas suas propriedades óticas ${ }^{[5-8]}$, reológicas $^{[9-15]}$ e nas morfologias resultantes ${ }^{[5-8]}$.

Thibault et al. ${ }^{[5]}$, por exemplo, estudaram as propriedades óticas em função das variáveis do processo de sopro, utilizando um LLDPE catalisado por Ziegler-Natta, z-LLDPE, um outro catalisado por metalocenos, mLLDPE, um LDPE e duas blendas de mLLDPE/LDPE. Estes autores verificaram um decréscimo nas opacidades e um aumento no brilho, exceto para o LDPE, com o aumento da razão de $\mathrm{D}_{\mathrm{r}}$ /BUR; observaram ainda que com o aumento desta razão, as estruturas dos filmes apresentavam textura fina, exceto o LDPE, que tinha textura grossa, associada à existência de lamelas cristalinas empilhadas. Os autores verificaram também que quanto maior a opacidade, maior era o diâmetro médio das estruturas do filme e que, a um dado $\mathrm{D}_{\mathrm{r}} / \mathrm{BUR}$, o diâmetro médio se comportava da seguinte forma: $\mathrm{mLLDPE}>$ LLDPE $>$ blendas $>$ LDPE.

Sukhadia et al. ${ }^{[7]}$ estudaram as origens das rugosidades superficiais e das opacidades em filmes soprados de m-LLDPE, z-LLDPE, catalisados por cromo, Cr-LLDPE, e de blendas de mLLDPE/Cr-LLDPE. Observaram que quando os polímeros apresentavam valores baixos de $\gamma_{\mathrm{r}}$ a opacidade dos mesmos era alta; este comportamento foi atribuído ao relaxamento rápido das moléculas levando à formação de estruturas cristalinas globulares, com alta rugosidade, e foi observado nos filmes de mLLDPE. Já quando os polímeros apresentavam valores intermediários de $\gamma_{\mathrm{r}}$, a opacidade diminuía, já que as moléculas tinham maiores tempos de relaxação, permitindo a formação de estruturas com alguma orientação, tipo "shishkebabs", com pouca rugosidade. Este comportamento era apresentado pelas resinas de $\mathrm{z}$-LLDPE. Finalmente a elevados valores de $\gamma_{\mathrm{r}}$ novamente a opacidade aumentava, devido a que os tempos de relaxação das moléculas eram muito elevados, formando-se estruturas com elevada opacidade e rugosidade. Neste caso, os efeitos elásticos derivados da extrusão (fratura de fundido, pele de cação) se sobrepunham à deformação recuperável. As resinas Cr-LLDPE se comportavam desta forma. Os mesmos comportamentos foram encontrados nas blendas de mLLDPE/Cr-LLDPE.

Fang et al. ${ }^{[9]}$ correlacionaram as propriedades reológicas (G', G" e razão Troutoniana, $\eta_{\mathrm{e}} / \eta_{\mathrm{o}}$, onde $\eta_{\mathrm{e}}=$ viscosidade 
Troutoniana e $\eta_{\mathrm{o}}=$ viscosidade do platô Newtoniano) com as variáveis do processo de sopro de duas resinas de LDPE, chamadas LDPEa e LDPEb, de uma resina de polietileno de alta densidade, HDPE, de um z-LLDPE e de um mLLDPE. Estes autores observaram que a estabilidade da bolha e o valor de G' tinham o mesmo comportamento: LDPEa e LDPEb $>$ HDPE $>$ LLDPE e mLLDPE. Assim concluiram que quanto maior G', maior a estabilidade da bolha durante o sopro.

Stark et al. ${ }^{[14]}$ estudaram o comportamento reológico de diversos polietilenos: um z-LLDPE, um mLLDPE e um LDPE. Observaram que as curvas de G'e G' do z-LLDPE tinham maior declividade do que as do LDPE, indicando que a distribuição de tempos de relaxação era muito mais larga em LDPE do que em LLDPE. Foi ainda observado que a viscosidade complexa, $\eta^{*}(\omega)$, era maior no LDPE do que no mLLDPE. A energia de ativação do fluxo, Ea, era maior para o LDPE, sendo este valor alto atribuído à presença de ramificações longas.

O objetivo deste trabalho foi, então, o de avaliar as propriedades reológicas de blendas de LLDPE/LDPE e correlacionálas com as propriedades óticas e a morfologia de filmes soprados destas blendas.

\section{Experimental}

\section{Materiais}

O LLDPE (comonômero buteno) e o LDPE foram fornecidos pela BRASKEM; os pesos moleculares, PM, e distribuições de pesos moleculares, DPM, de ambos polímeros foram obtidos por cromatografia de permeação a gel, GPC, num equipamento da Waters, modelo 150-CV, com colunas de Ultrastyragel, utilizando triclorobenzeno como solvente. A Tabela 1 mostra estes resultados.

\section{Sopro dos Filmes}

$O$ s filmes foram obtidos ${ }^{[3]} \mathrm{em}$ uma sopradora industrial marca Carnevalli CLD-75, com espessura do anel igual a 1,8 mm, diâmetro do parafuso igual a $75 \mathrm{~mm}, \mathrm{~L} / \mathrm{D}=24$, vazão mássica $\mathrm{Q}=90 \mathrm{~kg} / \mathrm{h}$ e $\mathrm{H}=30 \mu \mathrm{m}$. As composições LLDPE/LDPE: $100 / 0,90 / 10$ e $80 / 20$ foram processadas nas seguintes condições: $\mathrm{BUR}=3: 1, \mathrm{Z}=80 \mathrm{~cm}$, temperatura média do extrudado, $\mathrm{T}_{\mathrm{e}}=190{ }^{\circ} \mathrm{C}$ e $\dot{\boldsymbol{\varepsilon}}=\left(\mathrm{V}-\mathrm{V}_{\mathrm{o}}\right) / \mathrm{Z}=0,57$, para as blendas $100 / 0,0,52$ para as blendas $90 / 10$ e 0,51 para as blendas 80/20. Nestas condições, o LDPE puro não pode ser soprado.

\section{Caracterização reológica}

As blendas foram obtidas por extrusão de rosca simples, numa extrusora Guerst, com L/D $=30,5, \mathrm{~L}=61 \mathrm{~cm}, \mathrm{~T}_{\mathrm{e}}=210^{\circ} \mathrm{C}$

Tabela 1. Pesos moleculares do LLDPE e do LDPE obtidos por GPC.

\begin{tabular}{cccccc}
\hline Polímero & $\begin{array}{c}\mathbf{M}_{\mathrm{n}} \\
(\mathrm{g} / \mathbf{m o l})\end{array}$ & $\begin{array}{c}\mathbf{M}_{\mathrm{w}} \\
(\mathrm{g} / \mathbf{m o l})\end{array}$ & $\begin{array}{c}\mathbf{M}_{\mathrm{z}} \\
(\mathrm{g} / \mathbf{m o l})\end{array}$ & $\mathbf{M}_{\mathrm{w}} / \mathbf{M}_{\mathrm{n}}$ & $\mathbf{M}_{\boldsymbol{z}} \mathbf{M}_{\mathrm{w}}$ \\
\hline LLDPE & 8522 & 90328 & 264929 & 10,59 & 2,93 \\
LDPE & 7066 & 88357 & 295662 & 12,50 & 3,34 \\
\hline
\end{tabular}

e $\mathrm{Q}=6,8 \mathrm{~kg} / \mathrm{h}$, na forma de grãos. Estes grãos foram posteriormente prensados a $160^{\circ} \mathrm{C}$, durante $4 \mathrm{~min}$, a uma pressão $\mathrm{P}=100 \mathrm{kgf} / \mathrm{cm}^{2}$.

Todos os ensaios reológicos foram feitos às seguintes temperaturas: $190{ }^{\circ} \mathrm{C}, 200^{\circ} \mathrm{C}$ e $210{ }^{\circ} \mathrm{C} . \eta(\dot{\gamma})$ e $\mathrm{N}_{1}(\dot{\mathrm{Y}})$, a baixas taxas de cisalhamento, foram medidas num reômetro ARES da Rheometrics, de deformação controlada, com geometria cone-placa, ângulo de 0,01 radianos, e diâmetro de $25 \mathrm{~mm}$ e espaçamento entre as placas $h=0,048 \mathrm{~mm}$, sob atmosfera de nitrogênio, entre 0,01 e $100 \mathrm{~s}^{-1}$. Dos valores de $\eta_{\mathrm{o}}$ foi calculado a energia de ativação, $\mathrm{E}_{\mathrm{a}}$, utilizando a expressão de Arrehnius, ou: $\eta_{o}=A e^{E_{a} / R T}$, onde $\mathrm{A}=$ constante, $\mathrm{R}=$ constante universal dos gases e $\mathrm{T}=$ temperatura do fluxo $(\mathrm{K})$.

$\eta(\dot{\gamma})$ a altas taxas de cisalhamento foi medida num reômetro Capilar Instron 3211, utilizando um capilar de comprimento $L_{c}=30,4 \mathrm{~mm}$ e diâmetro $D_{c}=1,52 \mathrm{~mm}$, entre $10 \mathrm{~s}^{-1}$ até a taxa onde ocorreu fratura do fundido. Após as correções padrões (Rabinowisth e atrito do barril), os valores do índice de potencia, $n$, foram também calculados.

O inchamento do extrudado, $\mathrm{B}=\mathrm{D}_{\mathrm{e}} / \mathrm{D}_{\mathrm{c}}$, onde $\mathrm{D}_{\mathrm{e}}=$ diâmetro do extrudado, foi medido após resfriamento com nitrogênio líquido. Para cada extrudado, num comprimento de $5 \mathrm{~cm}$ foram feitas cinco medidas do diâmetro, utilizando um paquímetro. Os extrudados foram também analisados visualmente utilizando um estereoscópio, da marca DMI, com 40 $\mathrm{X}$ de aumento.

$G^{\prime}(\omega), G^{\prime \prime}(\omega)$ e $\gamma_{r}(t)$ foram medidos num reômetro de tensão controlada, AR-2000, da TA Instruments, utilizando geometria de placas paralelas, com $\mathrm{h}=1 \mathrm{~mm}$. Após a determinação do regime de viscoelasticidade linear, uma tensão de cisalhamento $\tau=500 \mathrm{~Pa}$, foi escolhida para a realização dos testes.

Para calcular $\gamma_{\mathrm{r}}(\mathrm{t}), \mathrm{a} \tau=500 \mathrm{~Pa}$ foi aplicada durante $300 \mathrm{~s}$, enquanto a deformação de cisalhamento, $\gamma$, era medida; após 300 s, a tensão era retirada e $\gamma$ era medida durante 600 s. $\gamma_{\mathrm{r}}(600)=$ deformação recuperável após $600 \mathrm{~s}$, foi calculada pela relação ${ }^{[16]}$ :

$$
\gamma_{r}(600)=\frac{\gamma(900 s)-\gamma(300 s)}{\gamma(900 s)} * 100
$$

onde $\gamma(900 \mathrm{~s})=$ deformação de cisalhamento após $900 \mathrm{~s}$ de início do teste,

e $\gamma(300 \mathrm{~s})=$ deformação de cisalhamento após 300s de início do teste.

\section{Propriedades óticas}

As opacidades total e interna dos filmes foram medidas de acordo com a norma ASTM D-1003, em um opacímetro da marca BYK-Gardner, modelo Haze Gard-plus. As opacidades totais foram medidas diretamente. Para medir as opacidades internas, as superfícies dos filmes foram umectadas com um líquido de índice de refração entre 1,51 e 1,54 (óleo de silicone), eliminando as opacidades superficiais. A opacidade superficial foi então obtida por meio da subtração da opacidade total da interna. $\mathrm{O}$ brilho foi medido num ângulo 
de $45^{\circ}$, segundo a norma ASTM 2457, no opacímetro acima descrito.

\section{Caracterização morfológica}

As superfícies dos filmes soprados foram analisadas num microscópio eletrônico de varredura, MEV, modelo Zeiss DSM 960 , com voltagem de $15 \mathrm{kV}$, após serem atacadas com permanganato de potássio em meio ácido, durante $20 \mathrm{~min}$, e recobertas com ouro. Os diâmetros das estruturas cristalinas formadas foram também medidos e suas médias calculadas em um programa de análise de imagens, IMAGE PRO-PLUS.

Para obter as rugosidades dos filmes, utilizou-se um microscopio de força atômica, Nano Scope $3 \mathrm{~A}$, com cabeçote de $100 \mu$ no modo contato.

\section{Determinação das cristalinidades "aparentes" dos filmes por difração de Raios $X$ de alto ângulo}

As \%'s de cristalinidade "aparentes" dos filmes foram medidas por difração de Raios-X utilizando um difratometro D5000 da Siemens, com radiação de CuK $\alpha$. Esta cristalinidade é considerada "aparente", porque inclui também a orientação molecular cristalina dos filmes.

\section{Resultados e Discussão}

\section{Caracterização reológica}

A caracterização reológica foi feita a três temperaturas, como descrito na parte experimental; porém mostraremos somente os resultados a $190^{\circ} \mathrm{C}$, já que os resultados a 200 e $210{ }^{\circ} \mathrm{C}$ seguiram o mesmo padrão que a $190{ }^{\circ} \mathrm{C}$. Os valores de $\eta(\dot{\gamma})$ a baixas e altas taxas de cisalhamento são mostrados na Figura 2. Observa-se que a viscosidade do LDPE puro é menor do que a do LLDPE puro e das blendas e que a adição do LDPE não afeta a resistência ao fluxo do LLDPE. Estes resultados estão de acordo com resultados anteriores. Os valores da viscosidade mostram a influência do tamanho das ramificações neste parâmetro: embora o LLDPE e o LDPE possuam DMP e PM próximos, o LLDPE é mais viscoso devido à presença das ramificações curtas. Em relação às

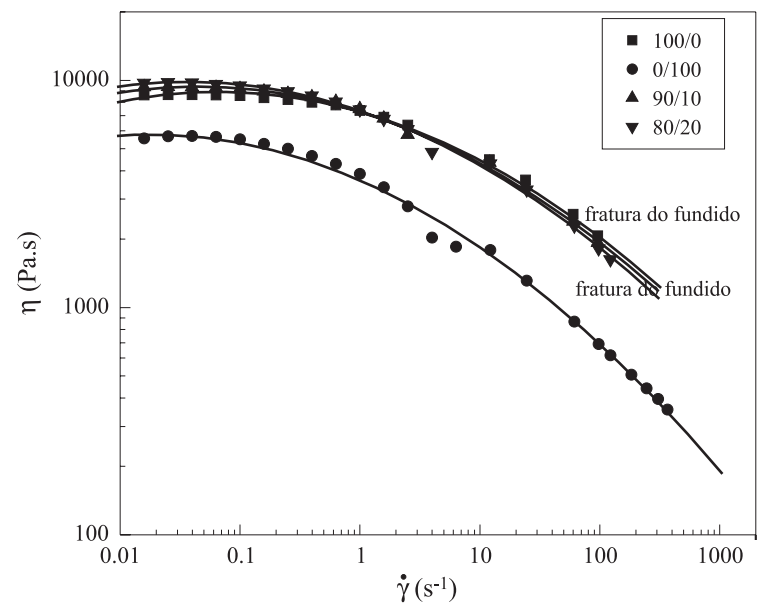

Figura 2. $\eta(\gamma)$
Tabela 2. $\eta_{0}$, n e deformação recuperável elástica, $\gamma_{\mathrm{r}}(600)$.

\begin{tabular}{ccccc}
\hline $\mathbf{T}\left({ }^{\circ} \mathbf{C}\right)$ & LLDPE/LDPE & $\boldsymbol{\eta}_{\mathbf{0}}(\mathbf{P a} . \mathbf{s})$ & $\mathbf{n}$ & $\begin{array}{c}\boldsymbol{\gamma}_{\mathrm{r}}(\mathbf{6 0 0}) \\
(\mathbf{\%})\end{array}$ \\
\hline \multirow{2}{*}{$\mathbf{1 9 0}$} & $100 / 0$ & 7529,35 & 0,55 & 0,713 \\
& $90 / 100$ & 5642,45 & 0,53 & 3,030 \\
& $80 / 20$ & 8909,30 & 0,54 & 0,765 \\
$\mathbf{2 0 0}$ & $100 / 0$ & 6376,62 & 0,56 & 1,081 \\
& $0 / 100$ & 4237,37 & 0,53 & 3,620 \\
& $90 / 10$ & 6853,51 & 0,56 & 1,290 \\
& $80 / 20$ & 7366,06 & 0,54 & 1,452 \\
\hline \multirow{2}{*}{$\mathbf{2 1 0}$} & $100 / 0$ & 5337,47 & 0,56 & 0,904 \\
& $0 / 100$ & 2856,76 & 0,51 & 6,830 \\
& $90 / 10$ & 5736,65 & 0,55 & 1,131 \\
& $80 / 20$ & 6315,70 & 0,53 & 1,107 \\
\hline
\end{tabular}

blendas, comportamentos diferentes foram encontrados $\mathrm{em}^{[12,13,17]}$, onde se observou que as blendas apresentavam viscosidades intermediárias entre as dos componentes puros. Das curvas de $\eta(\dot{\gamma}), \eta_{0}$ e $\mathrm{n}$ foram também calculados, como mostra a Tabela 2 . Observa-se que o valor de $n$ variou pouco com a adição do LDPE, ficando entre 0,56-0,51. Resultados semelhantes foram obtidos por Abrahan et al. ${ }^{[12]}$ nas suas blendas com LLDPE1/LDPE.

A Tabela 3 abaixo mostra os valores de $\mathrm{E}_{\mathrm{a}}$ das blendas.

A energia de ativação do LDPE foi maior que a do LLDPE,confirmando outros estudos ${ }^{[12,17,18]}$. O LDPE possui cadeias com ramificações longas e, se comparado com um linear de mesma estrutura, possui energia de ativação maior ${ }^{[18]}$. Este comportamento é resultante da presença destas ramificações longas, as quais em repouso, apresentam maior emaranhamento, necessitando-se então de maior energia para iniciar o fluxo. Quando o LDPE é adicionado ao LLDPE, a energia de ativação não se altera em relação ao valor do LLDPE puro. Os valores de $\mathrm{N}_{1}(\dot{\gamma})$ estão apresentados na Figura 3. Observa-se que o LLDPE puro é o menos elástico dos materiais quando em fluxo de cisalhamento e que a pequena adição de LDPE ao LLDPE torna as blendas muito mais elásticas, com valores próximos aos do LDPE puro.

$\mathrm{O}$ valor de $\mathrm{B}$ em função da taxa é mostrado na Figura 4 . O LLDPE teve o menor inchamento do extrudado, e o LDPE, o maior, comportamento similar ao da literatura ${ }^{[17]}$. Os inchamentos das blendas ficaram intermediários aos dos seus componentes puros.

Tabela 3. Energia de ativação das blendas de LLDPE/LDPE.

\begin{tabular}{ccccc}
\hline LLDPE/LDPE & $\mathbf{1 0 0 / 0}$ & $\mathbf{0 / 1 0 0}$ & $\mathbf{9 0 / 1 0}$ & $\mathbf{8 0 / 2 0}$ \\
\hline $\mathrm{Ea}(\mathrm{KJ} / \mathrm{mol})$ & 13,31 & 24,12 & 15,60 & 13,23 \\
\hline
\end{tabular}




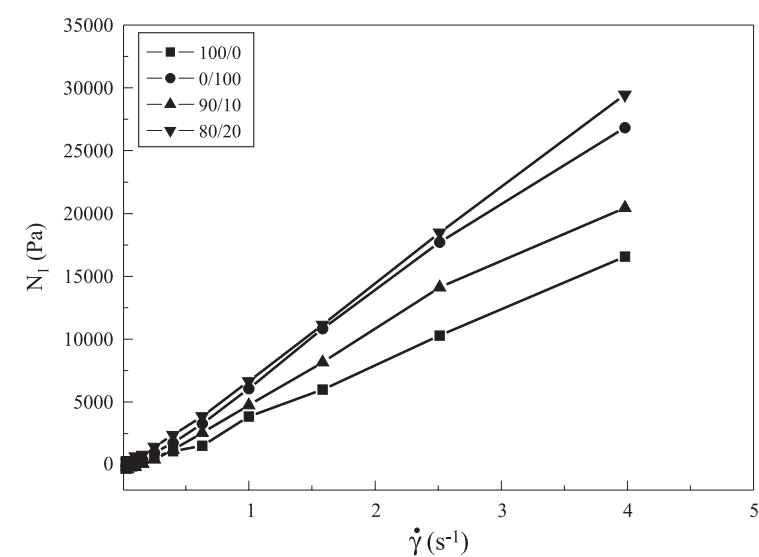

Figura 3. $\mathrm{N}_{1}(\dot{\gamma})$ das blendas de LLDPE/LDPE a $\mathrm{T}=190{ }^{\circ} \mathrm{C}$.

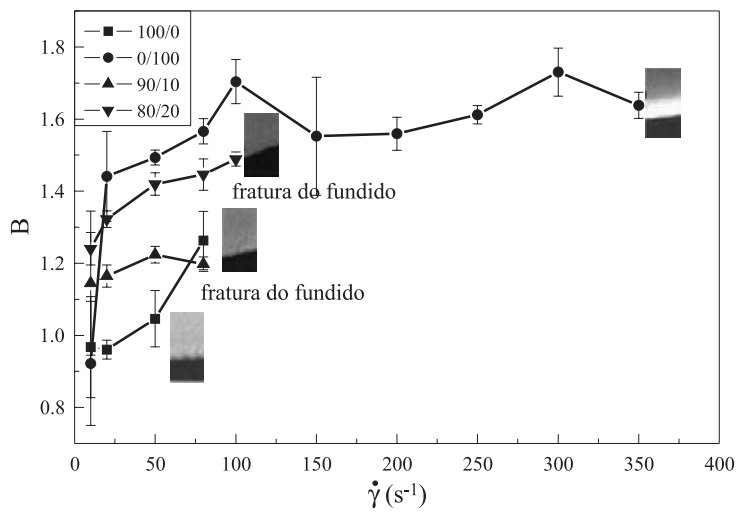

Figura 4. B a $\mathrm{T}=190{ }^{\circ} \mathrm{C}$ das blendas de LLDPE/LDPE.

O maior inchamento do extrudado do LDPE ocorre pela presença de ramificações longas. Estas ramificações ocupam maior volume livre; como o inchamento de extrudado tem influência da memória anterior ao fluxo, o LDPE inchará mais porque o seu estado conformacional anterior ao fluxo tinha maior volume livre que o do LLDPE. Os inchamentos das blendas são intermediários devido à presença do LDPE. Observa-se ainda que o inchamento do extrudado aumenta com o aumento da taxa de cisalhamento e diminui com o aumento da temperatura (não mostrado), comportamento observado em trabalhos anteriores ${ }^{[12,17]}$.

As taxas de cisalhamento críticas (início da fratura do fundido) das blendas são menores para o LLDPE puro e as blendas do que para o LDPE puro. A adição do LDPE ao LLDPE faz com que a fratura do fundido ocorra a taxas ligeiramente maiores nas blendas. As fotografias do estereoscópio são também mostradas na Figura 4. Observa-se que no LLDPE puro a fratura do fundido é mais irregular, e que esta irregularidade diminui com a adição do LDPE. As taxas de cisalhamento críticas aumentaram com a temperatura (comportamento não mostrado), retardando a fratura do fundido.

Os gráficos de $G^{\prime}(\omega)$ e $G^{\prime \prime}(\omega)$ das blendas são mostrados na Figura 5.

Pode-se observar que a freqüências baixas (equivalentes a taxas de cisalhamento baixas), o G' do LDPE é maior do que o do LLDPE e das blendas; porém a freqüências maiores,

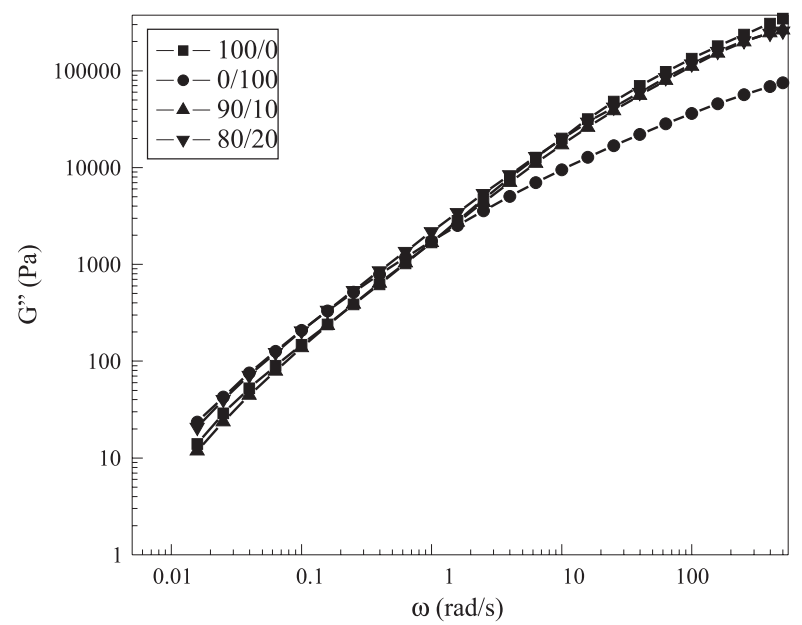

Figura 5a. Gráfico de G' para $\mathrm{T}=190{ }^{\circ} \mathrm{C}$ das blendas de LLDPE/LDPE.

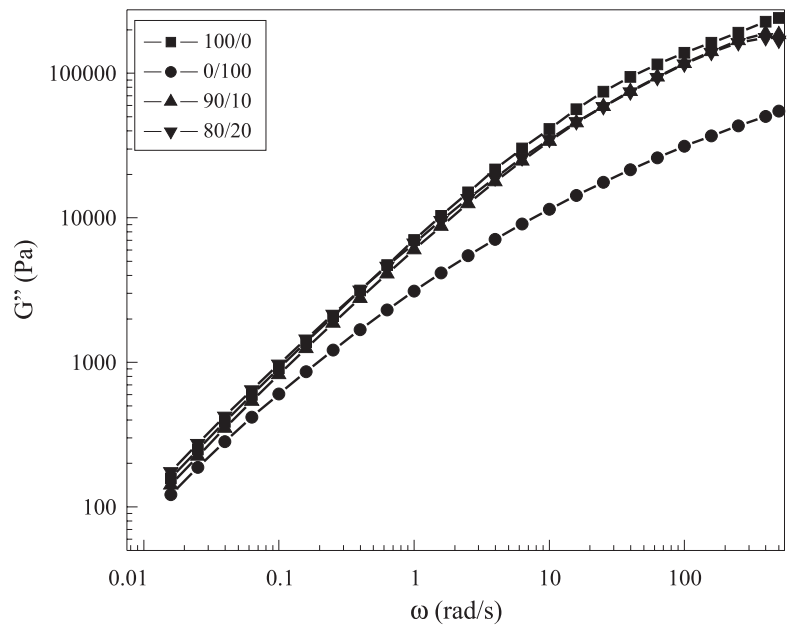

Figura 5b. Gráfico de G" para $\mathrm{T}=190{ }^{\circ} \mathrm{C}$ das blendas de LLDPE/LDPE.

observa-se o comportamento inverso. Quanto maior for G', maior for a estabilidade da bolha ${ }^{[9]}$, isto explica o fato do filme de LDPE puro não poder ter sido soprado nas condições impostas neste trabalho enquanto que as blendas e o LLDPE puro puderam ser soprados.

A Figura 6 mostra os ensaios de fluência e recuperação elástica das blendas e a Tabela 2 mostra os valores de $\gamma_{\mathrm{r}}$ calculados.

Da Tabela 2, conclui-se que o LLDPE puro relaxa mais rapidamente do que o LDPE e do que as blendas, ou seja, o LLDPE atinge mais rapidamente a sua deformação recuperável de equilíbrio, $\gamma_{\mathrm{r}}$. O tempo de relaxação pode ser correlacionado com o início da fratura do fundido. Kazatchkov ${ }^{[10]}$ observou que a taxa crítica aumenta, ou seja, a fratura do fundido aparece a taxas de cisalhamento mais altas, com o aumento do tempo de relaxação. Isso também pode ser comprovado neste trabalho: o valor de deformação recuperável para o LLDPE é menor, ou seja, o LLDPE relaxa mais rápido e com isso, a fratura do fundido aparece em taxas menores. Já com a adição de LDPE, os valores de recuperação elástica são maiores, ou seja, o processo de relaxação é mais lento do que no LLDPE puro, o que faz a fratura 


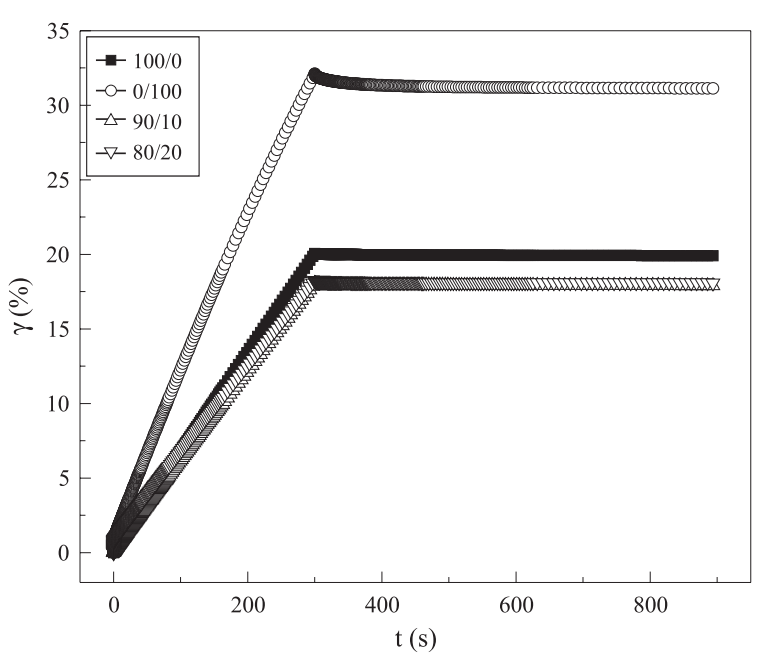

Figura 6. Deformação versus tempo, a $\mathrm{T}=190^{\circ} \mathrm{C}$, das blendas de LLDPE/ LDPE.

Tabela 4. Opacidades total, interna e superficial, brilho e espessuras dos filmes de LLDPE/LDPE.

\begin{tabular}{cccccc}
\hline $\begin{array}{c}\text { LLDPE/ } \\
\text { LDPE }\end{array}$ & $\begin{array}{c}\text { Opacidade } \\
\text { total } \\
(\boldsymbol{\%})\end{array}$ & $\begin{array}{c}\text { Opacidade } \\
\text { Interna } \\
(\boldsymbol{\%})\end{array}$ & $\begin{array}{c}\text { Opacidade } \\
\text { Superficial } \\
(\boldsymbol{\%})\end{array}$ & $\begin{array}{c}\text { Brilho } \\
(\boldsymbol{\%})\end{array}$ & $\begin{array}{c}\text { Espessura } \\
(\boldsymbol{\mu m})\end{array}$ \\
\hline $100 / 0$ & 48,20 & 10,80 & 37,40 & 21,10 & 56,88 \\
$90 / 10$ & 18,20 & 9,80 & 8,40 & 62,80 & 63,65 \\
$80 / 20$ & 10,00 & 5,10 & 4,90 & 79,30 & 63,00 \\
\hline
\end{tabular}

do fundido aparecer a taxas maiores. E o LDPE, com valores de recuperação elástica bem maiores que os outros materiais relaxa mais lentamente, e a fratura do fundido aparece então a taxas de cisalhamento ainda maiores.

Os resultados do brilho e das opacidades superficial, interna e total dos filmes de LLDPE/LDPE são mostrados na Tabela 4.

Observa-se que com o aumento da quantidade de LDPE nas blendas, as opacidades totais diminuíram, enquanto o brilho aumentou, já que este último varia inversamente com a opacidade superficial.

A correlação entre a opacidade total e a deformação recuperável está apresentada na Figura 7 , junto com as micrografias de MFA. Os valores de rugosidades médias determinados pela MFA estão na Tabela 5; e as estruturas dos filmes também analisadas por MFA estão na Figura 8.

Para o LLDPE puro, o valor de $\gamma_{\mathrm{r}}$ baixo resultou em um

Tabela 5. Rugosidades superficiais, diâmetros médios e cristalinidade aparente dos filmes de LLDPE/LDPE.

\begin{tabular}{ccccc}
\hline $\begin{array}{c}\text { LLDPE/ } \\
\text { LDPE }\end{array}$ & $\begin{array}{c}\mathbf{R}_{\text {média }} \\
(\mathbf{n m})\end{array}$ & $\begin{array}{c}\mathbf{D}_{\text {médio }} \\
(\boldsymbol{\mu m})\end{array}$ & $\begin{array}{c}\text { Cristalinidade } \\
\text { aparente } \\
(\boldsymbol{\%})\end{array}$ & $\begin{array}{c}\text { Cristalinidade } \\
\text { por DSC } \\
(\boldsymbol{\%})^{[3]}\end{array}$ \\
\hline $100 / 0$ & 117,17 & 5,60 & 39,72 & 15 \\
$90 / 10$ & 27,708 & 1,67 & 37,03 & 11 \\
$80 / 20$ & 23,427 & 1,08 & 35,43 & 9 \\
\hline
\end{tabular}

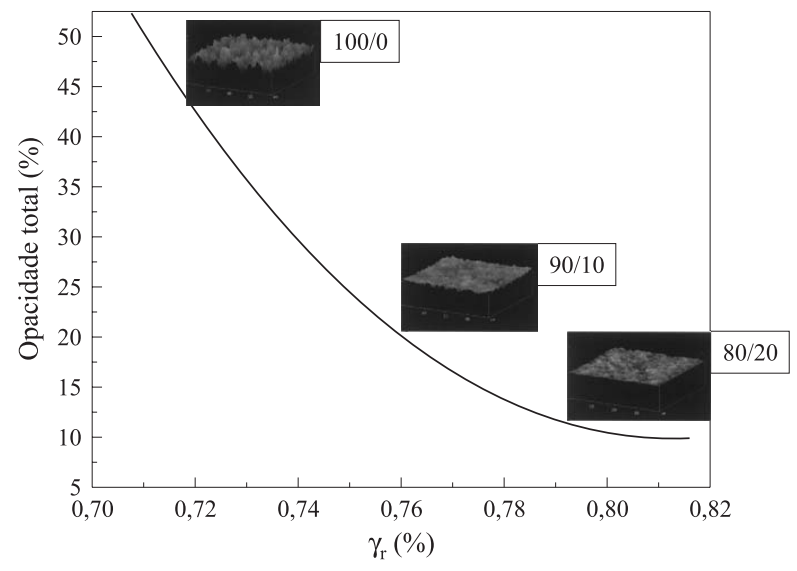

Figura 7. Correlação entre opacidades totais, deformações recuperáveis e superfícies dos filmes das blendas de LLDPE/LDPE.

tempo de relaxação curto, permitindo então que as moléculas cristalizassem rapidamente, sem a influência da tensão de elongação. Esta cristalização rápida, quase quiescente, permitiu a formação de estruturas esferulíticas grandes e grossas, e conseqüentemente o filme apresentou alta opacidade e maior rugosidade (Tab. 5). Já na blenda 90/10, o valor maior de $\gamma_{r}$ resultou em um tempo de relaxação mais longo que o primeiro; logo a cristalização ocorreu sob influência das tensões de elongação, fazendo com que se formem estruturas cristalinas pequenas, finas e orientadas. Conseqüentemente, os filmes destas blendas tiveram uma menor opacidade total e menor rugosidade média. Por último, na blenda $80 / 20, \gamma_{\mathrm{r}}$ foi ainda maior, o que resultou num tempo de relaxação ainda maior, e em estruturas cristalinas menores, finas e ainda mais orientadas, e valores menores de opacidade e rugosidade. Das micrografias de MFA, observa-se que as blendas apresentam maior orientação do que o LLDPE puro. As morfologias superficiais dos filmes podem ainda ser melhor visualizadas através das micrografias de MEV mostradas na Figura 9.

Observa-se ainda que o valor da cristalinidade "aparente" do filme de LLDPE puro é levemente maior do que o das blendas, seguindo a mesma tendência que os valores obtidos por DSC. Estes dados mostram então, que a \% de cristalinidade não é o único fator determinante das propriedades óticas do filme.

\section{Conclusões}

As seguintes conclusões podem ser retiradas deste trabalho:

$>$ Com a adição de LDPE ao LLDPE, houve aumento na viscosidade das blendas, resultando em resistências ao fluxo próximas às do LLDPE. A fratura do fundido ocorreu, porém, a taxas de cisalhamento mais altas. $\mathrm{O}$ mesmo comportamento foi observado com o aumento de temperatura. A estabilidade da bolha foi verificada através de G', sendo o menor valor encontrado para o LDPE e as blendas (menor estabilidade), e o maior valor para o LLDPE (estabilidade maior).

$>$ Uma correlação entre recuperação elástica, a opacidade total e a morfologia para as blendas de LLDPE/LDPE foi obtida: quanto maior a recuperação elástica, menor a opacidade total 


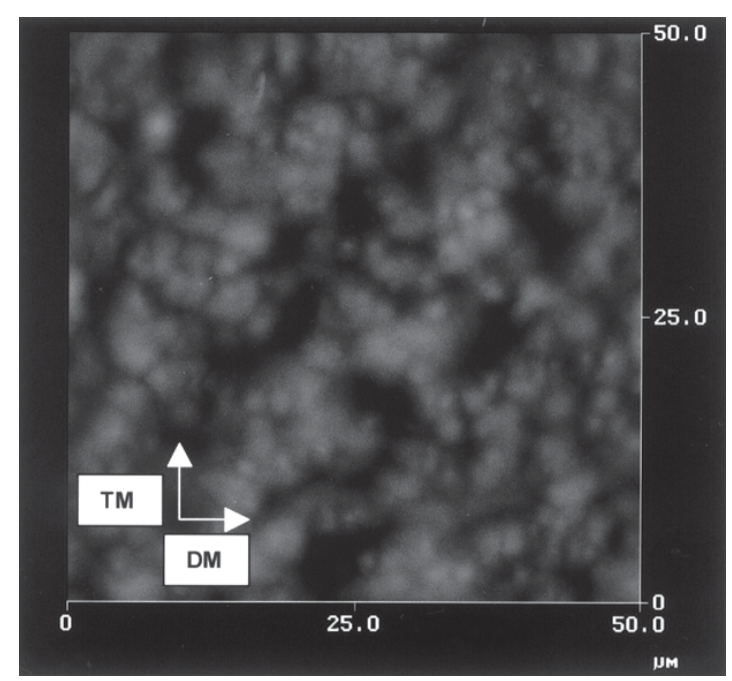

Figura 8a. Imagem da topografia do filme de LLDPE puro (Área $=50 \mu \mathrm{m}^{2} \mathrm{e}$ altura $=800 \mathrm{~nm}) . \mathrm{DM}=$ direção do estiramento vertical, $\mathrm{TM}=$ direção do estiramento circunferencial.

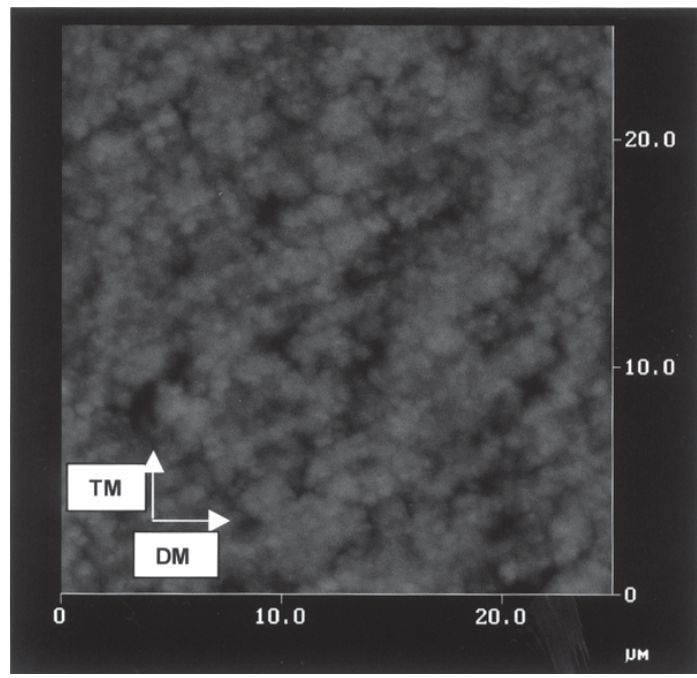

Figura 8b. Imagem da topografia do filme da blenda LLDPE/LDPE 90/10 (Área $=25 \mu \mathrm{m}^{2} \mathrm{e}$ altura de $500 \mathrm{~nm}$.)

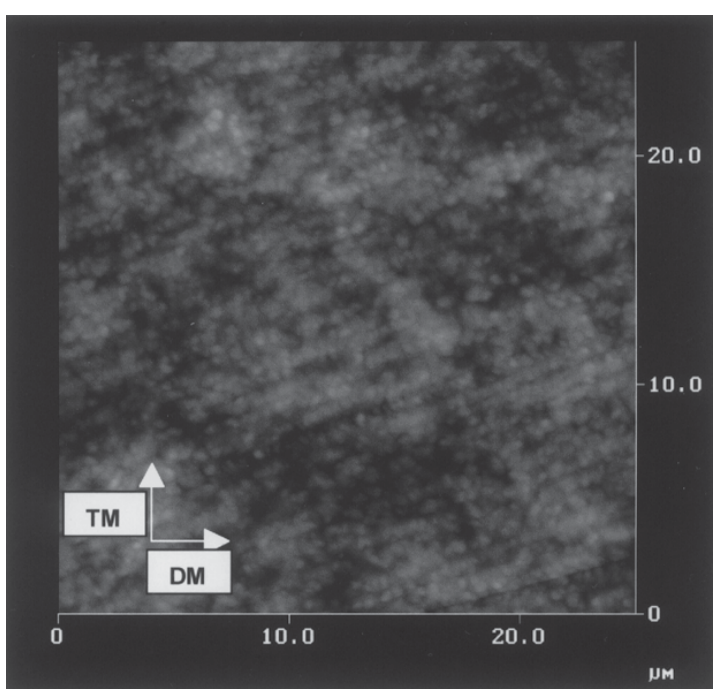

Figura 8c. Imagem da topografia do filme da blenda de LLDPE/LDPE 80/ $20\left(\right.$ Área $=50 \mu \mathrm{m}^{2}$ e altura de $300 \mathrm{~nm}$ ).

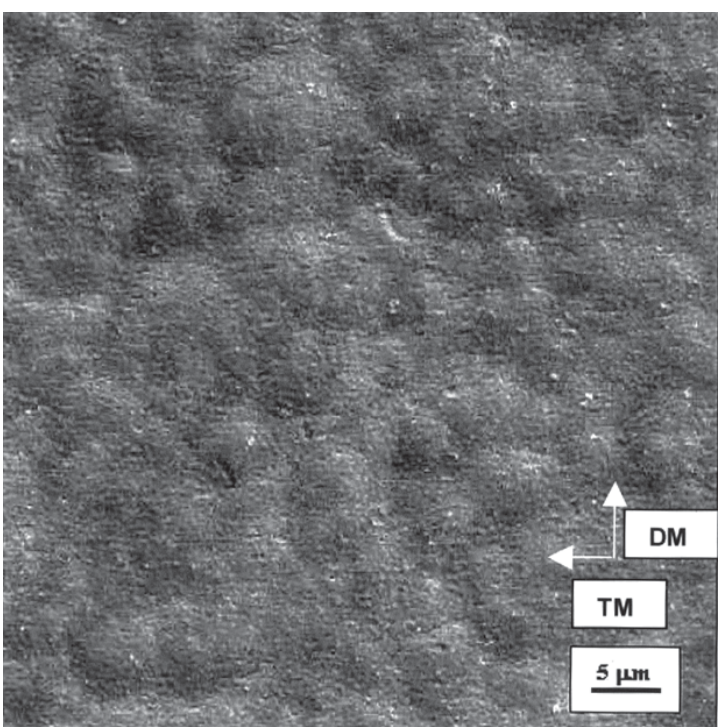

Figura 9a. MEV da blenda de LLDPE/LDPE, 100/0.

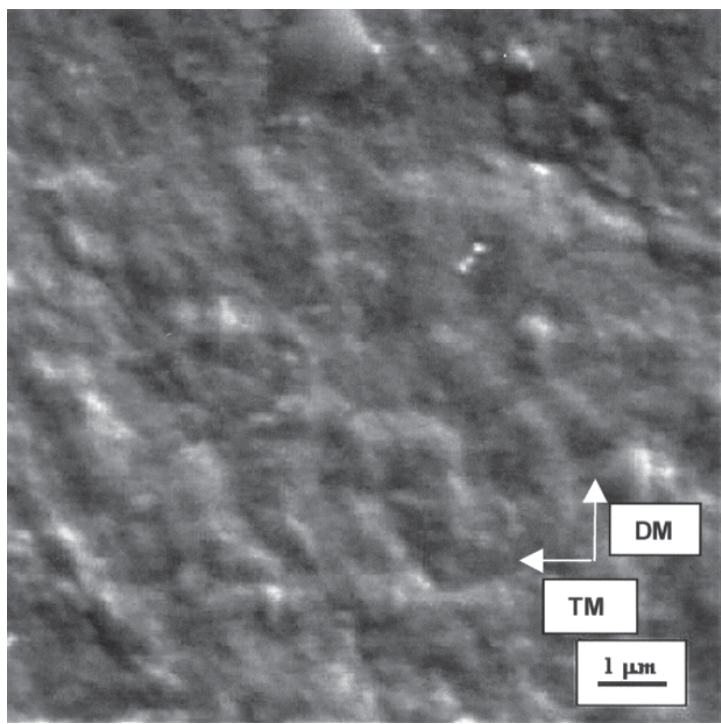

Figura 9b. MEV da blenda de LLDPE/LDPE, 90/10.

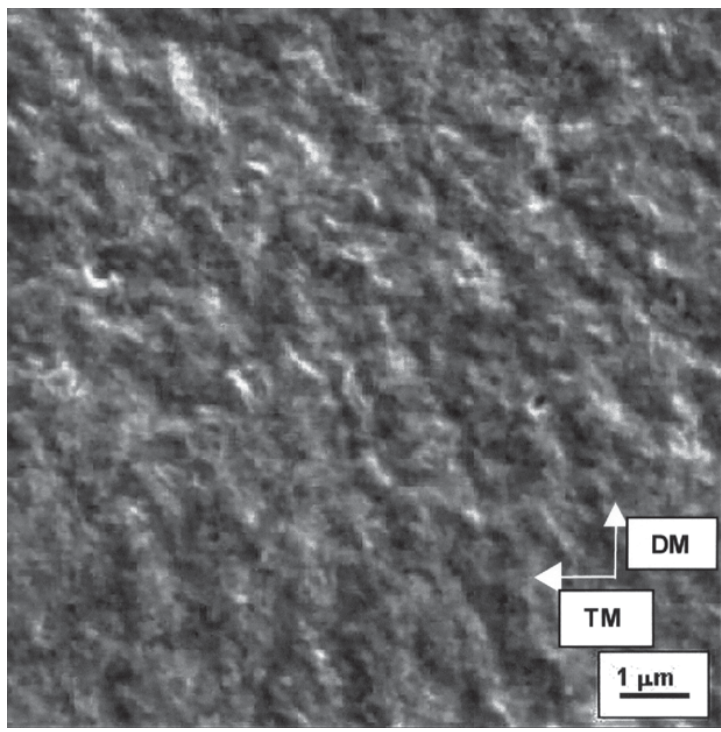

Figura 9c. MEV da blenda de LLDPE/LDPE, 80/20. 
e mais orientado e menor o cristalito. A adição do LDPE ao LLDPE aumentou a recuperação elástica das blendas diminuindo as suas opacidades e o tamanho dos cristalitos.

\section{Agradecimentos}

À Ruth C. Santana (UFSCar-DEMa) pelo processamento das blendas e à CAPES e FAPESP 00/06942-8 pelo financiamento deste projeto.

\section{Referências Bibliográficas}

1. Middleman, S. - "Fundamentals of Polymer Processing", McGraw Hill, New York, (1977).

2. Bretas, R. E. S., D’Avila M. A. -“Reologia de Polímeros Fundidos", EDUFSCar, São Carlos, (2000).

3. Pimentel, R. O. - "Correlação entre processamento e orientação molecular de filmes de polietileno produzidos por sopro", Dissertação, Universidade Federal de São Carlos, Brasil, (2001).

4. Peacock, A. J. - " Handbook of Polyethylene: Structure, Properties and Applications", Marcel Deker, New York, (2000).

5. Thibault, C.; Haudin J. M.; André J., M.; Ernest, B. "Morphological and crystallographic texture in polyethylene blown films. Influence on mechanical and optical properties", in: 17 th Annual Meeting of the Polymer Processing Society, CD-ROM, Montreal (2001).

6. Johnson, M. B.; Wilkes G. L.; Sukhadia A. M.; Rohlifing D. C. - J. Appl. Polym. Sci., 77, p. 2845, (2000).

7. Sukhadia, A. M.; Rohlfing, D. C.; Johnson M. B.; Wilkes, G. L. - J. Appl. Polym. Sci., 85, 11, p. 2396, (2002).
8. Jordens, K. - "The influence of small amounts of $L D P E$ on the morphology and resulting haze of LLDPE blown films", in: Annual Technical Conference of the Society of Plastics Engineers, CD-ROM, San Francisco, (2002).

9. Fang, Y.; Carreau, P. J.; Lafleur, P. G. - "Rheological effects in film blowing”, in: 17 th Annual Meeting of the Polymer Processing Society,CD-ROM, Montreal, (2001).

10. Hussein, A. J.; Willians, C. M. - Polym. Eng. Sci., 41, 4, p. 696, (2001).

11. Kazatchkov, I. B; Bohnet, N.; Goyal, S. K.; Hatzikiriakos, S. G. - Polym. Eng. Sci., 39, 4, p. 804, (1999).

12. Abrahan D.; George K. E.; Francis D. J. - J. Appl. Polym. Sci., 62, p. 59, (1996).

13. Becker, M.; Forte, M. M. C.; Baumhardt $\mathrm{N}^{\text {to }}$. R. B. Polímeros: Ciência e Tecnologia, 12, 2, p. 85, (2002).

14. Stark, P.; Malmberg, A.; Löfgren B. - J. Appl. Polym. Sci., 83, 5, p.1140, (2002).

15. Yamaguchi, M.; Abe, S. - J. Appl. Polym. Sci., 74, 13, p. 3160, (1999).

16. Silva L.; Bretas R. E. S. - Polym Eng. Sci., 40, 6, p. 1414, (2000).

17. Utracki, L. A.; Shlund B. - Polym. Eng. Sci., 27, 5, p 367, (1987).

18. Dealy, J. M., Wissbrun K. J., - "Melt Rheology and Its Role in Plastics Processing", London: Chapman \& Hall, (1995).

Recebido: 04/09/03

Aprovado: 17/12/03

\section{O arquivo disponível sofreu correções conforme ERRATA publicada no Volume 14 Número 2 da revista.}

